\title{
REGULATORY STRATEGY FOR REGISTRATION OF COMBINATION PRODUCTS TO
}

\section{US-FDA}

Available online at www.ijdra.com

\section{REVIEW ARTICLE}

\author{
KothaVihar*,Elphine Prabahar, Rama Rao Nadendla, Desu Bhrama Srinivasarao \\ Chalapathi Institute of Pharmaceutical sciences, Guntur, Andhra Pradesh \\ *Corresponding Author's E-mail: vihar000@gmail.com
}

\begin{abstract}
In pharmaceutical industry all the healthcare products are categorized into Drugs, Devices, and biologics and they are regulated by their respective departments of the regulatory authorities. Due to more and more development of science and technology in the current scenario, had led to the invention of innovative novel products in healthcare system for better diagnosis and treatment of diseases, advancement of these novel technologies have led to a the blurring of historical lines of separation between the healthcare products, so these advances had led to the existence of combination products.

Combination products raise a variety of regulatory and review challenges compared with single-entity products. They have the potential to provide enhanced therapeutic advantages. It ranges in complexity from Drug-eluting stents to gene therapy systems and from chemotherapeutic Drugs combined with monoclonal antibodies to novel nanotechnology-based Drug delivery systems. They include innovative products for diagnostic and therapeutic treatments of cardiovascular, metabolic, and oncologic disorders, among other types of products.

In this article we describe the regulations for registration of combination products in US and their regulatory considerations for identification, jurisdiction and review, premarket activities, applicability of Good Manufacturing Practices (GMPs) and post marketed requirements, adverse event reporting, inspection and enforcement.
\end{abstract}

Keywords: OCP, PMOA, USFDA, CDER, CDRH, CBER.

\section{INTRODUCTION}

Imagine a nanoparticle-monoclonal antibody combination, that when exposed to light, emits toxic oxygen radicals that kill cancer. A photo activated polymer combined with autologous cartilage to tissue engineer a new knee joint. Are these products Drugs or Devices? How should they be approved and regulated. Combination products are those Nano platforms, micro machines, and other bioengineered Drugdelivery systems coupled to a biotechnologically crafted molecule or protein, will continue to change how we diagnose and treat disease. These products, like follow-on biologics, will create issues and opportunities for those who develop, use, and, pay for them. (1)

An estimated $30 \%$ of all new healthcare products under development today are combination products. Why? Because Drugs, biologics and medical Devices, when used alone, may not be sufficient to treat disease or injury. To tackle the clinical problems of the future, these products will be combined to treat a wide range of diseases from heart attack and stroke to Alzheimer's, cancer, and diabetes and beyond. In fact, every area of medicine will be benefitted because damage of disease or injury potentially can be erased, not just stopped

\section{COMBINATION PRODUCT MARKET}

It was estimated that combination products market is increased from approximately US\$6 billion in 2004 to nearly $\$ 10$ billion by 2009 . Furthermore, the total global value of the DrugDevice combination products market is increases to $\$ 11.5$ billion in 2010. Currently, the market has a potential of US\$20.6 billion and has been experiencing a growth of $15 \%$ CAGR for the past two years. In the next five years, the market is expected to grow at a CAGR of $11.8 \%$. global Drug Device combination products market expected to grow to $\$ 115.1 \mathrm{~b}$ in 2019. A future view of the Drug-Device combination is that these products will make single component medical Devices obsolete. (2) 
Untapped market potential and benefits are the primary factors for the early adoption. The other market factors contributing are relatively shortened product approval time with the establishment of Office of Combination Products (OCP) within the FDA. An increase in the number of cardiovascular patient population has also facilitated the early acceptance of the Drug-Device combination products. This is supported by the fact that cardiovascular applications constitute about $64 \%$ of the entire Drug-Device combination applications market. However, it is the relatively smaller market of antimicrobial catheter but that offers the highest growth potential over the next five years in the overall market.

The Drug-Device combination market is not fragmented and the key players in the this market are Medtronic, Boston Scientific Corp., Edwards Life sciences Corp., Stryker Corp., QLT Inc. etc The maximum number of new product developments is expected to take place in the bone graft substitutes, advanced wound care products and antimicrobial catheter markets.

\section{Definition of combination product (3):}

A combination product is defined as:

a) A product composed of two or more regulated components, i.e., Drug/Device, biologic/Device, Drug/biologic or Drug/Device/biologic, that are physically, chemically or otherwise combined or mixed and produced as a single entity.

b) Two or more separate products packaged together in a single package or as a unit comprised of Drug and Device products, Device and Biological products or Biological and Drug products.

d) Any investigational Drug, Device or Biological product packaged separately that according to its proposed labeling is for use only with another individually specified investigational Drug, Device or Biological product where both are required to achieve the intended use, or effect.

e) Drug- Drug, Device-Device, or biologicbiologic products do not meet the definition of a combination product.

FDA'S Office of Combination Products OCP:
The task of designating a lead agency center for review and regulation of combination products was assigned to the Office of the Chief Mediator and Ombudsman in the Office of the Commissioner. Over the years, a number of concerns have been raised about combination products, including concerns about the consistency, predictability, and transparency of the assignment process; issues related to the management of the review process when two or more FDA centers had review responsibilities for a combination product; and lack of clarity about the post market regulatory controls applicable to combination products.

FDA's Office of Combination Products (OCP) was established on December 24, 2002, as required by MDUFMA, to address such concerns. The law gives OCP broad responsibilities covering the regulatory life cycle of combination products. As outlined in MDUFMA, the responsibilities of OCP include:

$>$ Assigning an FDA center to have primary jurisdiction for review of a combination product

$>$ Ensuring timely and effective premarket review of combination products by overseeing reviews involving more than one agency center.

$>$ Ensuring consistency and appropriateness of post market regulation of combination products.

$>$ Resolving disputes regarding the timeliness of premarket review of combination products.

$>$ Updating agreements, guidance documents, or practices specific to the assignment of combination products and

$>$ Submitting annual reports to Congress on the office's activities and impact.

\section{COMBINATION PRODUCTS AND US REGULATIONS:}

\section{PRIMARY MODE OF ACTION (PMOA)} (4):

The FDA defines mode of action as the means by which a product achieves its intended therapeutic effect, in which "therapeutic" action or effect includes any effect or action of the combination product intended to diagnose, cure, 
mitigate, treat, or prevent disease or affect the structure or any function of the body.

Combination products are composed of more than one type of regulated product (Biological product, Device, or Drug) and each part contributes its mode of action, combination products will have more than one mode of action. So The FDA defines the primary mode of action PMOA as "the single mode of action of a combination product that provides the most important therapeutic action of the combination product." The most important therapeutic action is the one expected to make the greatest contribution to the overall intended therapeutic effects of the product.

Typically, combination products with a PMOA attributable to the Drug component are assigned to the CDER, a Device PMOA to the CDRH, and a biologic PMOA to the CBER (21 CFR 3.4). The assigned center will consult or collaborate with the other centers during the review of the combination product. Once assigned to a lead center, the combination product will typically follow that center's application type for premarket review.

For example: Most Drug delivery systems are combination products consisting of both a Drug and a Device. They may be simply a syringe prefilled with a Drug or a complex iontophoretic Drug-delivery patch. Usually, the most important therapeutic action of such a product is attributable to the Drug component's role in treating a disease and the Device plays a secondary role in delivering the Drug. These types of products are reviewed by the CDER, with the CDRH consulting on the Device aspects of the product.

\section{REQUEST FOR DESIGNATION:}

There may be issues when the PMOA of a combination product cannot be identified with certainty or when a product has two or more distinct modes of action and neither is subordinate to the other. In those cases, the developers will write a request for FDA to assign the PMOA of the product.

To start the process, a company will submit a "Request for Designation" to the (OCP) office of combination product where a determination will be made concerning the primary mode of action after consultation with the appropriate Centers. Based on this determination, the product will be assigned to one Center as the lead for the review of the premarket application and regulation. During the review of the premarket application, the Centers will confer and consult with each other so that the review, evaluation, and regulation of the product reflect the combined evaluation of the appropriate Centers.

\section{PRODUCT JURISDICTION:}

A decision algorithm is used to assign the combination product to a lead center (21 CFR 3.4(b)). The FDA first assesses whether there is a center that regulates other combination products that present similar questions of safety and effectiveness with regard to the combination product as a whole and, if so, assigns the product accordingly.

When there are no other combination products that present similar questions for safety and effectiveness, the agency assigns the combination product to the center with the most expertise in evaluating the most significant safety and effectiveness questions presented by the combination product. Once assigned to a lead center, an investigational new Drug or an investigational Device exemption application are submitted. Following the investigational period, a single marketing application should be ensured for product safety and effectiveness, as well as to ensure consistent and appropriate post market regulation for most combination products.

For combination products being developed by more than one manufacturer, there may be a desire to provide information to the FDA while maintaining the confidentiality of each manufacturer's intellectual property. The application holder can accomplish this by submitting to the FDA a letter of authorized cross-reference from the owner of the referenced material. In some instances, two marketing applications may also be appropriate. The product developers should discuss with the FDA for two marketing applications to their specific product. (4) 
Table 1: Comparison of regulatory aspects of Drug and Devices

\begin{tabular}{|c|c|c|}
\hline & Drugs & Devices \\
\hline Regulated by: & CDER & CDRH \\
\hline Relevant sections & $\begin{array}{l}\text { Drug cGMPs ( } 21 \text { CFR } \\
210-211)\end{array}$ & Device cGMPs (21CFR 820) \\
\hline Emphasis & $\begin{array}{l}\text {-Quality systems } \\
\text { - Corrective and } \\
\text { preventive action } \\
\text { - Individual outputs } \\
\text { - Large clinical trials } \\
\text { - Risk assessment (but } \\
\text { not really...) }\end{array}$ & $\begin{array}{l}\text {-Quality systems } \\
\text { - Design controls } \\
\text {-Quality by design } \\
\text { - Risk assessment } \\
\text {-Process as a continuum } \\
\text { - Small, focused clinical } \\
\text { trials (maybe) }\end{array}$ \\
\hline Timeframes & Long (6-10 years) & Short-medium ( $1-5$ years) \\
\hline
\end{tabular}

$$
\begin{aligned}
& \text { CDER = FDA Center for Drug Evaluation and Research } \\
& \text { CDRH = FDA Center for Device and Radiological Health } \\
& \text { CFR = Code of Federal Regulations } \\
& \text { cGMPs = Current good manufacturing practices }
\end{aligned}
$$

\begin{tabular}{|c|c|c|}
\hline LEAD CENTER & APPLICATION TYPE & REVIEW CLOCK \\
\hline CDER/CBER LEAD & $\begin{array}{l}\text { New Drug Application or } \\
\text { Biologic License Application }\end{array}$ & $\begin{array}{c}6 \text { Month (Priority Review) } \\
\text { Or } 10 \text { Month (Standard } \\
\text { Review) }\end{array}$ \\
\hline \multirow[t]{3}{*}{ CDRH } & Pre Market Approval & 180 Day \\
\hline & $\begin{array}{c}510 \mathrm{~K} \\
\text { premarket notification }\end{array}$ & 90 Days \\
\hline & $\begin{array}{l}\text { HDE } \\
\text { humanitarian device } \\
\text { exemption }\end{array}$ & 75 Days \\
\hline
\end{tabular}

Table 2: Applications for combination products according to PMOA and review period 


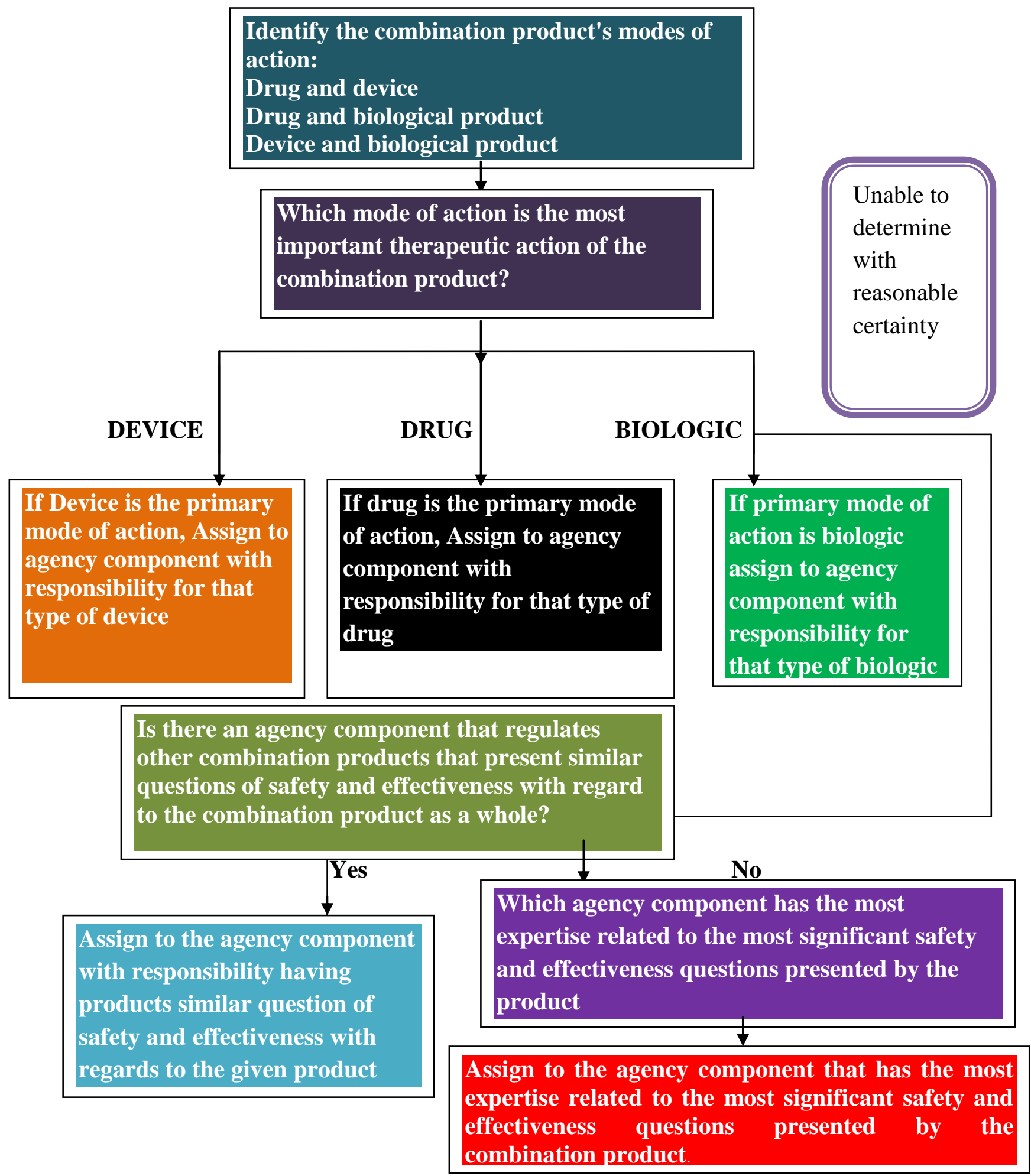

Figure 1: Decision algoritham for assignment of lead center

\section{PRODUCT APPROVAL PROCEDURE:}

After the assignment of lead centre the product developer can approach the lead center for market authorization by following the required market application to the lead center.
If the lead centre is CDER then the product should be submitted under NDA and if the product is assigned to CBER then the application is BLA similarly if the lead center is $\mathrm{CDRH}$ then it is applied as 510k or PMA depending on the classification of the Device. 
For example: A combination product with Device and Drug components is the Gem 21S dental bone graft with growth factor. The primary purpose of this product is the repair of periodontal defects. The secondary action is for the Drug component to promote growth of new bone. Since the primary mode of action is derived from the Device component, the product is regulated by the CDRH as a Device, and it would be reviewed through a PMA application.

Another example with a different outcome would be the transdermal patch. The primary purpose of the patch is for the treatment of ADHD while the patch itself is merely a delivery system for the Drug. Therefore this product is regulated by CDER as a Drug under a New Drug Application (NDA).

\section{Drug and Device Development and Approval Process (5):}

To obtain Device approvals, the first step is to determine the classification of Devices under class I, II, or III and to develop the data (e.g., biocomparability, pre-clinical and clinical data). Depending on the classification of Devices, different processes, ranging from Pre-Market Notification of Class II Devices to IDE and PreMarket Approval (PMA) for class III Devices, are utilized. These approved Devices are required to be properly labeled, registered and listed. Also, as a part of approval process, GMP inspections by FDA using quality system will take place at the manufacturing site. In general, the time required for product development, even with the class III Devices, clinical investigation and approval of Devices is shorter and it is a less expensive process relative to the development of Drugs or biologics.

Development of Drugs in general is a much longer and more expensive process involving many different stages from research and development, pre-clinical, and clinical studies. Human clinical studies are further divided into phase 1 , phase 2 , and 3 with different purposes and study durations and scales. These stages are designed to evaluate the product from initial safety and dosing determination to efficacy. The initial stage starts with candidate selection, synthesis, and purification, followed by the animal testing, which would take up to approximately 18 months. The second stage of human clinical studies, starting from the phase 1 studies in healthy subjects to phase 2 and 3 in patient populations, takes somewhere between 2 and 5 years. Finally, submission and review of NDA/BLA for the marketing approval normally would require from 6 to 10 months.

Post-marketing surveillance, adverse event reporting and submission of post-approval changes are parts of the continuing regulatory oversights by FDA after marketing approval.

\section{Clinical considerations for combination products (6):}

Industry stakeholders should consider for the development of combination products that may challenge existing approaches to progress from a novel concept to an innovative marketed product For example:

$>$ What pre-clinical or animal studies are appropriate?

$>$ What types of clinical trial designs may be appropriate?

New methodologies may need to be developed for manufacturing, evaluation of preclinical safety in targeted areas of the body, and clinical trial design to establish safety and effectiveness. Many combination products consist of a Device and a Drug or a Biological product. The purpose of the Device component in such a combination may provide the primary use, with the other product serving an adjunctive use. In other cases, the Device may serve the secondary role as a vehicle for delivery of the Drug or biologic. In either case, compatibility between the various products must be tested. For timed-release usage, elution testing will be evaluated.

\section{Device constituent part:}

Device constituent parts that are already approved for another purpose, the extent of preclinical testing largely would focus on the new use of the Device constituent as part of the combination product.

If a combination product incorporates an indwelling, intravenous Drug delivery catheter for a new use for long-term, Drug delivery in the brain, new biocompatibility studies may be necessary to establish the safety of the Device materials for placement in neural tissues. New engineering or functional testing may also be 
necessary to establish the suitability of the Device design to the new environment in which it will be used.

\section{It may be appropriate to conduct studies to} evaluate the following potential risks:

Leachables/extractables of the Device materials into the Drug/biologic substance or final combination product.

$>$ Changes in stability of the Drug constituent when delivered by the Device or when used as a coating on the Device.

$>$ Drug adhesion/absorption to the Device materials that could change the delivered dose.

Presence of inactive breakdown products or manufacturing residues from Device manufacture that may affect safety, or Device actions that could change the Drug performance characteristics at the time of use.

$>$ Change in the stability or activity of a Drug constituent when used together with an energy emitting Device.

$>$ The material properties of a delivery Cather may be adversely affected by Drug/biologic products.

\section{Drug and Biological product constituent part:}

When new molecular entity (NME) is a constituent part of a combination product, it is critical to consider the information necessary to characterize the safety and effectiveness of the NME when used in the combination product.

When the combination product contains a Drug/biologic constituent that is already approved for another use, then the development should address the safety and effectiveness of the new combination product.

The following additional preclinical or clinical safety information is appropriate for the Drug/biologic constituent and the combination product:

$>$ Approved Drug or Biological product with a change in formulation, strength, route of administration or delivery method.

D New dosage e.g., absolute dose, dosing duration, dosing regimen, or total exposure.
$>$ New patient population, (e.g., pediatric, geriatric, pregnant or nursing women, or change in disease or disease status).

$>$ Change in approved indication.

Other possible requirements for development of a product containing Drug/Device include:

$>$ In vivo pharmacokinetic studies may be necessary to assess changes in formulation, strength, route of administration, dosing, population or other factors that may alter the extent or time course of systemic exposure. These studies might be used to determine Drug release kinetics such as release rate, local peak concentrations of the Drug, local distribution and systemic bioavailability $\mathrm{C}_{\max }, \mathrm{T}_{\max }$, etc.

$>$ Dose ranging or dose finding studies in humans may be appropriate to determine dose adjustments for safety/effectiveness when therapy is targeted to a local site.

$>$ Acute and repeat dose toxicity studies using the new route of administration may be appropriate to determine the NOAEL (no observed adverse effect level) and toxicity profile of the combination product. Typically, these studies would evaluate the intended clinical formulation and dosing regimen/frequency that will closely approximate its use in clinical settings.

$>$ Special safety studies may be appropriate for certain patient populations e.g., hepatotoxicity, QT prolongation, special populations.

$>$ Specific safety monitoring in the clinical study may be appropriate to obtain data on the novel aspects presented by the combination product; e.g., local toxicity for a new route of administration.

\section{CLINICAL INVESTIGATION}

All Drugs must prove safety and efficacy prior to marketing, although they are not required to prove benefit over existing therapies. A similar requirement exists for most class III Devices that undergo the PMA process. However, most Devices enter the market through the less rigorous $510(\mathrm{k})$ process where they, at most, need only to show equivalence to a predicate Device, indicating that the Device does what it is intended to do and is reasonably safe. Demonstration of efficacy is not required for 
approval. It is important that clinicians are aware of these differences in premarketing scrutiny and take them into consideration when selecting treatment for a patient.

For most combination products

$>$ Investigational New Drug (IND)

$>$ Investigational Device Exemption (IDE)

Any of the two is submitted for the clinical investigation of the combination product as a whole.

Generally, the regulatory guidance for INDs and IDEs provides substantial flexibility in considering how to address the issues posed by a particular product.

There are two guidance's to combination products are:

(1) Exploratory IND Studies

(2) Changes that may occur during investigational development of a Device.

\section{Human factor studies:}

Combination products that include a Device constituent part, it may be necessary to evaluate the human factors of Device use on the safety and effectiveness of the combination product:

To demonstrate that the users can safely and effectively use the injector in subsequent pivotal clinical studies, you should perform a comprehensive evaluation of all user related risks, and as needed, conduct human factors/usability studies with the final version of the Device to validate users' performance with representative users. Depending upon the characteristics of the Device, its intended user population, and the environment of use, the studies should focus on essential aspects of using the product. These aspects may include the ability of users:

- To read, understand and follow instructions

- To adequately set up the Device

- To reconstitute injectable materials, draw up or set the proper dose

- To perform the injection or self-injection correctly; and

- To perform disposal of sharp components and other materials safely.
Addition user related factors are:

- How users operate the system in realistic \& stressful conditions

- Controls

- Displays

- Software

- Logic of operation

- Labels \& instructions

- Analysis of critical tasks

- Use error hazard and risk analysis

Human factors evaluations take place early in the combination product development process to identify design features that may need modification before conducting the key studies to establish the safety and effectiveness of the combination product.

\section{Sterility requirements (7):}

Sterility is critical if the Device is intended for sterile use or the Device is intended for use in sterilizing other products. The sterilization methods such as steam, ethylene oxide, gamma radiation, and so on, will be important in determining the test method and validation point. The testing should provide results for the sterility assurance level (SAL).

Manufacturers of medical Devices must validate processes, including Sterilization, for a Device purporting to be sterile. Stability testing should be part of design validation of such Devices when a product is labeled as sterile it is considered to be stability characteristic and must be tested for shelf life. The sterility testing should take into account the mechanical performance of the Device, the integrity of the packaging, the shipping and transportation methods, and any other environmental factors that could affect the sterility of the Device up to the time of use.

\section{Stability requirements (5):}

The purpose of stability testing is to provide evidence on how the quality of a substance or product varies with time under the influence of a variety of environmental factors such as temperature, humidity, and light. Such testing enables the manufacturer to establish or modify recommended storage conditions, retest periods, and shelf life or dating period. Shelf life testing 
is important for products subject to degradation overtime. In addition to the factors mentioned above, the length of shelf life may, especially for long-term implantable Devices, depend on the specific bodily fluids with which the Device may come into contact shelf life should be adequately supported and substantiated by real time testing.

\section{DEVICE BIOCOMPATIBILITY STUDIES CONDUCTED DURING PREMARKET APPROVAL OF COMBINATION PRODUCTS}

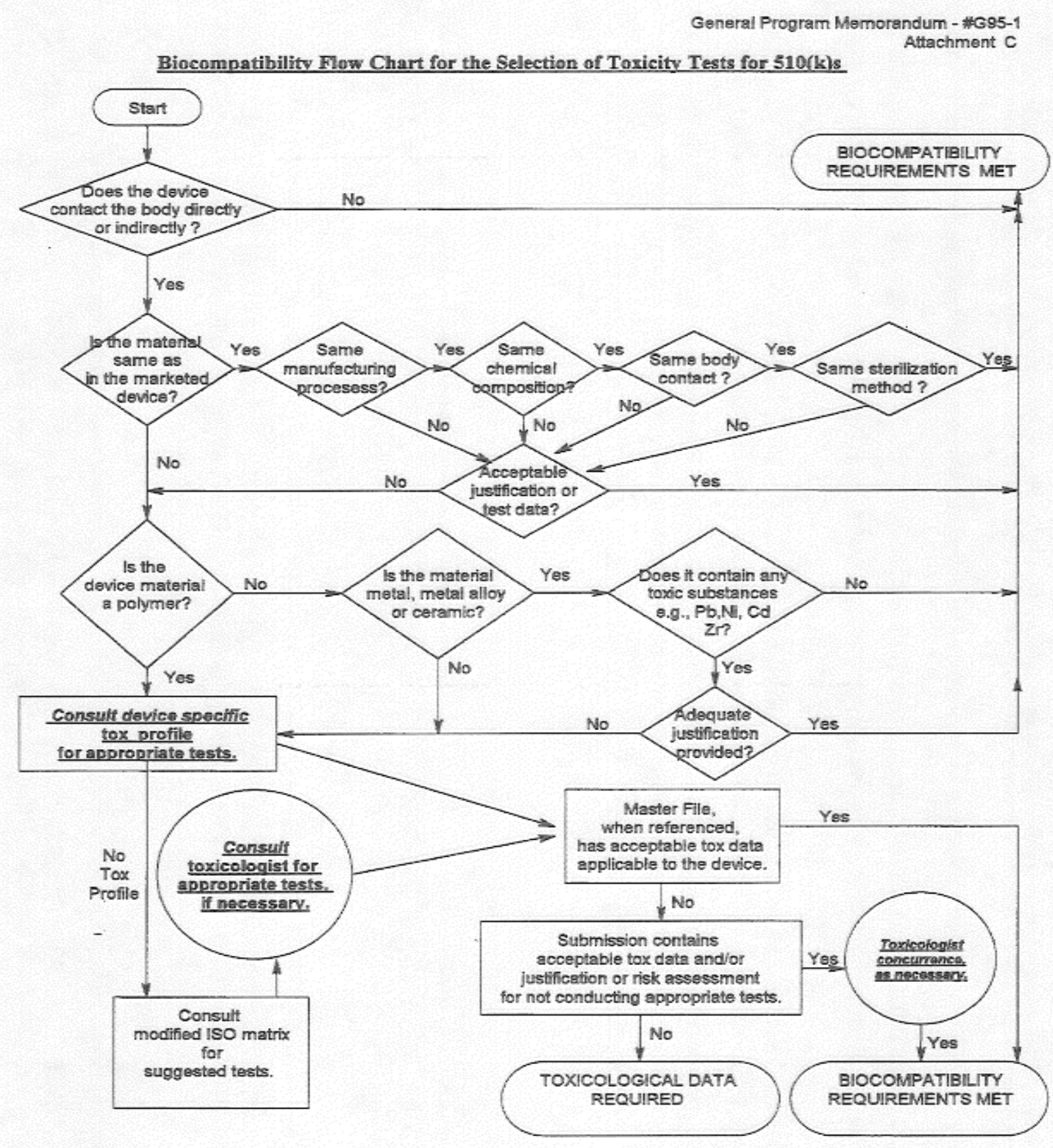

Figure 2: Device Biocompatibility Studies conducted during premarket approval of Combination Products

The manufacturers may not be familiar with the regulatory requirements for one part of combination product due to differences in approval process under different laws and regulations. Specifically, adequate stability testing of Drug/Device combinations to satisfy these requirements can be a difficult task. Without adequate stability testing, the integrity 
of Drug/Device combinations may not hold up during manufacturing, storage and transport or during use, resulting further delay of product approval It is important to conduct stability studies properly to assure the stability of these combinations, starting from the early stages of Drug testing and up to the post-marketing studies to assure its performance.

For Drug component in a combination product, stability testing generally follows $\mathrm{ICH}$ guidelines (e.g., ICH Q1A). In comparison, requirements for stability testing on Devices are very different. For example, aging studies of a Device are not covered under current ICH guidance for Drugs and biologics. Nevertheless, to assess the stability of a combination product, the tests should cover both Drug and Device components and the following issues need to be considered during development:

- Stability data requirements and shelf-life determination.

- Storage and shipping conditions.

- Device aging studies with the presence of Drug components.

- Stability of carrier components for a Drug, if applicable.

- Compatibility between Drug and Device components.

- Leachables and extractables during storage.

- Effects of manufacturing process on product stability.

- Sterilization process and product stability.

- In-use stability including mechanical stress.

- Stability testing and manufacturing changes.

- Reserve samples for stability testing.

There are also a few unique items which required special attentions during the design of a stability program for a Drug-Device combination. First, a shorter development cycle of a Drug-Device combination requires the final stability protocol to be developed early to collect sufficient data to support the final product approval. Second, unlike a traditional Drug product, a combination product such as Drug-eluting stent is typically manufactured at a small lot size, therefore matrixing and bracketing designs for stability testing are necessary to avoid consuming a large number of samples for the stability testing.

Finally, stability indicating tests are determined by:

(1) In vivo and in vitro release rate for the Drug component,

(2) Stability of critical inactive ingredients (such as integrity of coating polymers),

(3) The interactions between the Drug and the Device,

(4) Effects of sterilization process on the product, and

(5) Monitoring of fatigue, corrosion, durability of the Device part.

However, for the approval of a PMA for a product such as Drug-eluting coronary stent, it is necessary to collect stability data from the final product containing the final Device design and formulation in the marketing package manufactured at the commercial production site and scale. The expiration dating of the products needs to be established based on the stability data. Also, stability data collected under stress or in-use conditions may be needed for the final product approval.

Unlike the approval of Devices for which expiration dating generally is not needed, an expiration dating supported by stability data is absolutely required for a Drug-Device combination product. It is advised to design a stability program early enough to meet the short development timeline and to avoid frequent changes in the product and process.

The stability indicating tests should be developed to cover unique characteristics of both Drug and Device components in a combination product and assess the effects on stability by the interactions between the two components. Also, it is a good idea to apply bracketing/matrixing and skip-lot approaches to reduce the sample size required for stability testing

\section{General CMC Issues for Combination Products:}

For product approval, chemistry, manufacturing and controls (CMC) requirements for Drug component in a combination product are similar to those for Drug substance and Drug product in 
a stand-alone Drug product. However, additional data relevant to the unique characteristics of a combination product also need to be considered for regulatory approval. Typically, CMC information for a combination product should cover the following areas:

$>$ Drug substance and formulation.

$>$ Product characterization.

$>$ Manufacturing process and in-process controls.

$>$ Release specifications for products.

$>$ Relationships of Drug and mechanical function, durability or reliability of a Device's components.

$>$ Effects on Drug stability by Device components.

$>$ Potential leaching of other components.

$>$ Degradation products resulting from breakdown of the biomaterials from Device components.

$>$ Device impact on rate of release and absorption of Drug.

$>$ Drug stability in the combination products.

\section{LABELING (8):}

Products that are designated as combination products through labeling present challenges and Opportunities. Under 21 CFR 3.2(e) (3) of the FD\&C Act, a new Drug, Device or biologic product intended for use with an already approved, individually specified regulated product may be a combination product when the labeling of the already approved product "needs to be changed" upon approval of the new product. This is known as a "cross labeling" or "mutually conforming labeling" issue.

For example, suppose a sponsor of a unique intravenous delivery Device wishes to label the Device for use with a Drug product that is currently labeled for subcutaneous administration. Under the regulations, if the labeling of the Drug would "need to be changed" upon approval or clearance of the new delivery Device, the two products would be considered a combination product. If the two products are considered a combination product, then, in all likelihood, the primary mode of action of that product would be attributable to the Drug component, and the product would be assigned to CDER for review and regulation. This would result in the new Drug delivery
Device also being reviewed by CDER under the new Drug provisions of the act.

Alternatively, there may be instances when a sponsor of a new product intended for use with an already approved product would prefer his/her product to be a combination product. For example, suppose the sponsor of a new Drug intended it to be used with an already marketed Device, and that the two products were considered a combination product, with the primary mode of action of the product being attributable to the Device component. In such a case, the sponsor of the new Drug might argue that the Device labeling would need to be changed to reflect the use of the Drug with the Device, thereby resulting in $\mathrm{CDRH}$ being designated as the lead agency centre for reviewing and regulating the new Drug. Such an approach would require that the sponsor of the Device agreed to amend its product's labeling upon approval of the new Drug.

\section{GMP CONSIDERATIONS (9):}

The Food, Drug, and Cosmetic Act recognizes combination products as a category of medical products distinct from Drugs, biologic products and medical Devices, which are the constituent parts of combination products.

The current Good Manufacturing Practice (cGMP) for combination products regulation will be referred to as the combination products quality system regulations. The quality system regulation that applies to Drugs and biologics are $(21$ CFR 210, 211) referred to as cGMP. The quality system regulation that applies to medical Devices (21 CFR 820) is referred to as the QSR. The cGMP and QSR regulations are also referred to as predicate rules.

The final rule incorporated as constituent parts in combination products. And Current Good manufacturing Practice states that cGMP regulations for Drugs and biologic products apply to combination products that include a Drug or biologic constituent part. cGMP regulations for medical Devices or the QSR, apply to combination products that include a Device constituent part. Additional regulatory requirements and standards apply if certain Biological products described in 21 CFR 600680 are requirements, including donor eligibility 
requirements, for human and cellular and tissuebased products.

There are two options for demonstrating compliance with applicable quality system requirements for a single-entity or a copackaged combination product containing both a Drug or biologic and a medical Device. A company may demonstrate compliance with the specifics of all quality system regulations applicable to each constituent part or under certain conditions; it may demonstrate compliance with the specifics of either of the predicate rules rather than both.

In order to demonstrate full compliance with both regulations, a manufacturer basing its quality system on a cGMP platform also may be required to demonstrate compliance with specified provisions of the QSR, thus creating a streamlined (i.e., hybrid) quality system. These specified provisions are:

$>$ Management responsibility

CFR 820.20)

$>$ Design controls (21 CFR 820.30)

$>$ Purchasing controls (21 CFR 820.50)

$>$ Corrective and preventive action (21 CFR $820.100)$

> Installation (21 CFR 820.170)

> Servicing (21 CFR 820.200)

A combination product manufacturer choose to base its quality system operations on the QSR platform, the manufacturer also must demonstrate, as applicable, compliance with specified provisions of the cGMP regulation, again forming a streamlined quality system these specific provisions are as follows:

$>$ Testing and approval or rejection of components, Drug product containers and closures (21 CFR 211.84)

$>\quad$ Calculation of yield (21 CFR 211.103)

$>$ Tamper -evident packaging requirements for over the-counter (OTC) human Drug products (21 CFR 211.132)

$>$ Expiration dating (21 CFR 211.137)

$>$ Testing and release for distribution (21 CFR 211.165)

> Stability testing (21 CFR 211.166)

$>$ Special testing requirements (21 CFR 211.167)

> Reserve samples (21 CFR 211.170)
When two or more types of constituent parts to be included in a single-entity or co-packaged combination product are held at the same facility, or when the manufacture of a combination product proceeds at the same facility while utilizing these constituent parts, compliance with all applicable quality system requirements must be demonstrated.

For the development or manufacture of a particular combination product, when a conflict occurs between individual predicate rule requirements, the final rule directs that the regulations most specifically applicable to the constituent part at issue take precedence over the more general requirement.

The final rule mainly addresses single-entity and co-packaged (i.e., kit) combination products. It clarifies that certain container closure systems that also serve as Drug delivery Devices (e.g., a prefilled syringe) may be considered Drug manufacturing components but are still constituent parts of combination products and subject to the final rule. Therefore, if a facility manufactures a finished prefilled syringe from Drug and Device components, it must comply with both QSR and cGMP regulations.

Regardless, if the manufacturer of a Devicecontaining combination product chooses to establish its quality system on a cGMP or QSR platform, the design history file requirements of design controls must be satisfied. The manufacturer must address all design issues resulting from the combination of constituent parts and demonstrate that the combination product was developed in accordance with a prospectively established design plan. FDA's Office of Combination Products stated that design controls apply not only to the Device constituent part of a combination product but also to the overall combination product, possibly including certain aspects of the Drug constituent part.

For example, for a co-packaged combination product, the compatibility of the constituent parts should be assessed using design controls, in particular if the Drug constituent part is optimized for use in combination with a particular Device. 
For investigational combination products being developed under an Investigational New Drug (IND) application or Investigational Device Exemption (IDE), in certain circumstances, FDA allows such products to be exempt from certain quality system requirements. Combination products containing a Drug or Biological product, which are in Phase 2 or Phase 3 clinical studies, are subject to the final rule. Investigational medical Devices are exempt from all of the QSR requirements, except design controls.

The preamble also briefly discusses how the final rule applies to cross-labeled combination products. Because the constituent parts of a cross-labeled combination product are manufactured and marketed separately, they remain separate for purposes of applying the predicate rules. The constituent parts of a crosslabeled combination product still must be manufactured in accordance with the quality system requirements that would apply if they were not part of a combination product.

The final rule does not change any quality system requirements described in predicate rules for constituent parts (i.e., Drug, biologic, Device) described in master files (e.g., Drug Master Files and Master Files for Devices). Under the final rule, if the manufacture of an article described in a master file is subject to cGMP or QSR requirements, these requirements must still be met. If the manufacture of such an article is exempt from certain predicate rule requirements, it may still be subject to other predicate rule requirements (e.g., QSR purchasing controls in the case of Device constituent parts).

\section{POSTMARKETING SURVEILLANCE (10):}

FDA has not issued a regulation covering the reporting of adverse events for combination products. The agency has however, issued for comment purposes only, a concept paper on post market safety reporting for combination products.

It considered the fact that each constituent part of a combination product is governed by one of three differing sets of reporting provisions. The agency reviewed each set of regulations governing post marketing safety reporting for
Drugs 21CFR part 310 and 314, Biological products 21 CFR parts 600 and 606, and Devices 21 CFR part 803.

In general, each set of regulations requires reports of death and serious adverse events; each provides periodic and follow-up reports; and each provides a method to signal certain types of safety events that warrant expedited reporting. Because of these similarities, it is possible to consolidate the requirements so that the combination product is subject primarily to the reporting requirements associated with the type of marketing application under which the product is approved. However, there are certain significant differences in the three sets of regulations. These differences are designed to facilitate adverse experience reporting that adequately addresses the distinct characteristics and potential safety issues related to a particular type of product (i.e., Drug, Device, and Biological product). The public health benefit of these unique provisions would be lost if the combination product were subject solely to the reporting requirements associated with the type of marketing application. FDA has identified five such provisions, unique to Drugs, biologics, or Devices, that need to be preserved to appropriately reflect the combination nature of the product and to ensure consistent and appropriate post marketing safety reporting for combination products:

\section{5-Day Report:}

The Medical Device Reporting (MDR) regulation has a provision found in $\S 803.53$ (a), which requires reporting no later than five work days after the day the reporter becomes aware that an MDR reportable event associated with the Device necessitates remedial action to prevent an unreasonable risk of substantial harm to the public health. This section also allows FDA to make written requests for the submission of all subsequent events of the same nature that involve substantially similar Devices for the time period specified in the written request. Reporters must also maintain a record of any report they submit under this provision. This provision is unique to Devices; a similar provision is not found in the Drug or Biological product reporting regulations. 


\section{0-Day Device Malfunction Report:}

The MDR regulation also includes $\S 803.20$ (b) (3) (ii), which requires reporting no later than 30 calendar days after the day the reporter becomes aware of information that reasonably suggests the Device has malfunctioned and that this Device or a similar Device that the reporter markets would be likely to cause or contribute to a death or serious injury if the malfunction were to recur. Reporters must also maintain a record of any report they submit under this provision. Like the 5-day MDR report, this situation is unique to Devices, and the Drug and Biological product reporting regulations do not have comparable provisions.

\section{5-Day “Alert Report" for Drugs and Biological Products:}

A reporter must submit to FDA a report of an adverse experience associated with the use of a Drug or Biological product that is both serious and unexpected, whether foreign or domestic, as soon as possible but in no case later than 15 days of initial receipt of the information as set forth in $\S \S 314.80$ (c) (1) and (e), and 600.80(c) (1) and (e). Serious events are reportable within 30 days under $\S 803.20$ (b) (3) (i) for Devices, regardless of whether or not they are expected. However, there is no requirement in the MDR regulation for expedited (15-day) reporting of an event that is both serious and unexpected.

\section{3-Day Field Alert Report:}

Another unique provision is $\S 314.81(\mathrm{~b})(1)$, which requires applicants to file "field alert reports" when there is information concerning certain types of problems with a Drug in distribution, such as any bacteriological contamination, or any significant chemical, physical, or other change or deterioration in a distributed Drug product, or any failure of one or more distributed batches of the Drug to meet the specification established for it in its marketing application, or any incident that causes the Drug product or its labeling to be mistaken for, or applied to, another article. Reporters must submit this information to the FDA district office that is responsible for the facility involved within 3 working days of its receipt. They must provide the information by telephone or other rapid communication means, with prompt written follow up. Reporters must also maintain a record of any report they submit under this provision. These types of situations are specific to Drug products, and neither set of regulations found in parts 600 (Biological products) or 803 (Devices) has a similar provision requiring expedited submission of these types of reports.

\section{Expedited Blood Fatality Report}

Section 606.170 requires expedited reporting of a complication of blood collection or transfusion confirmed to be fatal, by telephone, facsimile, express mail or electronically transmitted mail as soon as possible and a written report within 7 days after the fatality.

Reporters must also maintain a record of any report they submit under this provision. This situation is specific to blood products. Although parts 310, 314, 600 and 803 require expedited reporting of deaths, they do not provide for the immediate notification of blood related fatalities.

\section{POST APPROVAL CHANGES (10):}

The type of submission to provide for the change will depend on the type of application used to obtain approval of the combination product. The submission is independent of the change in the constituent part. For example if the combination product submission is to CDER the post approval changes is submitted as PAS or CBE-30 and in the case of CDRH it is submitted as 180-day supplement or PMA 30day notice.

\section{CDER/CBER:}

Prior approval supplement for NDA/BLA

CBE 30

\section{CDRH:}

180-day Supplement

PMA 30-day Notice

\section{CASE STUDY FOR CLASSIFICATION OF COMBINATION PRODUCTS(11):}

Although classification as Drug or medical Device can be very clear for some products, for some other products it is not so obvious. Some products that may intuitively be considered a Drug may, in fact, actually be classified as a Device, and vice versa. It is important to 
remember that the classification of a product is determined by its PMOA for combination products. Here given some case studies for determination of PMOA.

\section{CASE STUDY 1}

After the formation of the $\mathrm{OCP}$, a retrospective review of existing combination products was conducted to determine which center should assume primary responsibility for review and regulation. One of the more notable decisions that resulted from this review was the reclassification of heparin flushes from Drug to Device. In the announcement of transfer, the FDA stated that heparin flushes exert their PMOA by physically occupying space and applying pressure within the catheter, similar to the mechanism of saline flushes. The mechanism of heparin preventing thrombotic occlusions was determined to be a secondary function of the product. Both saline and heparin flushes are now classified as class II Devices requiring $510(\mathrm{k})$ clearance for marketing. This change surprised many health care providers because heparin is considered a high-alert medication. They may not be aware that, as class II Devices, saline and heparin flush solutions only had to demonstrate substantial equivalence to a Device already on the market to obtain FDA clearance.

\section{CASE STUDY 2}

Topical creams used to treat minor dermatologic conditions are commonly thought to be Drugs. However, some topical creams are considered to be barriers and are classified as Devices because they impart no chemical or metabolic action and have no active ingredients. Tropazone CR (Midlothian Laboratories) is one example of a prescription-only cream used for the management of superficial wounds and first and second degree burns. This emulsion contains moisturizers that work to keep the area moist and was approved through the $510(\mathrm{k})$ process, showing technological comparisons to 4 predicate Devices. Clinical testing involved only insult patch testing in 50 human subjects, showing it to be a non primary irritant or skin sensitizer. No efficacy studies showing benefit to the healing process were reported in the 510(k) application.

\section{CASE STUDY 3}

Formulation of a product may also affect its classification as Drug or Device. Oral sucralfate (Carafate) acts chemically with hydrochloric acid in a patient's stomach to form a barrier paste inside the body, thereby creating a protective barrier at ulcer sites. The FDA classifies oral sucralfate as a Drug because it acts chemically within the body to perform its action. In contrast, sucralfate topical paste (Carapaste) is mixed with hydrochloric acid prior to use, forming a paste that is then applied to oral lesions. The resulting product acts physically as a protective barrier and is classified as a Device.

\section{CONCLUSION}

US regulations for combination products were created to ease difficulties arising in the product jurisdiction of combination products in which either of the three centers CDER, CDRH, CBER, having responsibility over one product. While the FDA has little discretion over the assignment of a lead agency centre based on the primary mode of action of the product. There are other areas where flexibility and creativity are possible with combination products. One should have a clear idea for the assessment of primary mode of action of the product to make feasible for the invention, development, manufacture and approval of combination product.

\section{ACKNOWLEDGEMENT}

I express my gratitude to my family members for their support throughout my work. It's my immense pleasure to express my deep sense of gratitude and sincere thanks to Mr. K.S. Babu \& Mr. Vasu for their valuable suggestions and continuous encouragement to complete my work successfully.

\section{CONFLICT OF INTEREST}

Author declares that there are no conflicts of interest.

\section{REFERENCES}

1. Manresa H, and Arleen D. Meyer's combination products and the FDA: issues and answers. Biotechnology healthcare [Internet]. 2005 Feb[Cited 2014 May 13]. Available from: 
http://www.ncbi.nlm.nih.gov/pmc/articles/PMC3 564323/

2. Lauritsen KJ, Nguyen T. Combination Products Regulation at the FDA. Clinical pharmacology \& Therapeutics [Internet]. 2009 May[Cited 2014 Jun 11]. 85(5). Available from:

http://www.nature.com/clpt/journal/v85/n5/full/c lpt200928a.html

3. Mark D. Kramer Combination Products: Challenges and Progress. Regulatory Affairs Focus [Internet]. 2005 Aug [Cited 2014 May 1]. Available from:

http://www.fda.gov/downloads/CombinationProd ucts/MeetingsConferencesWorkshops/UCM1167 23.pdf

4. Siew A, Navigating Landscape of Combination Products. Pharmaceutical Technology Europe [Internet]. 2014 April [Cited 2014 June 26]. Available from: http://www.pharmtech.com/pharmtech/Formulati on/Navigating-the-Regulatory-Landscape-ofCombination/ArticleStandard/Article/detail/8397 05

5. Huynh K B. Handbook of Stability Testing in Pharmaceutical Development. New York, NY 10013, USA: Springer Science Business Media; 2008.

6. Guidance for Industry and FDA Staff Early Development Considerations for Innovative Combination Products. Office of Combination Products (OCP). [Internet]. 2006 Sept [Cited 2014 June 3]. Available from:

http://www.fda.gov/RegulatoryInformation/Guid ances/ucm126050.htm

7. Lewis A. Drug-Device Combination Products Delivery Technologies and Applications. 1st edition. Wood head Publishing Limited and CRC Press LLC; 2010.

8. Suzanne O'Shea Working through the US Rules for Combination Products. The regulatory affairs journal. [Internet]. 2008 Oct [Cited 2014 May 5]. Available from:

http://www.faegrebd.com/webfiles/Working\%20 Through $\% 20$ the $\% 20$ U.S.\%20Rules $\% 20$ for $\% 20 \mathrm{C}$ ombination $\% 20$ Products.pdf

9. Current Good Manufacturing Practice for Combination Products Guidance for Industry and FDA. Office of Combination Products (OCP). [Internet]. 2004 Sept [Cited 2014 May 7]. Available from:

http://www.fda.gov/regulatoryinformation/guida nces/ucm126198.htm

10. Guidance for Industry and FDA Post marketing Safety Reporting for Combination Products. Office of Combination Products (OCP). [Internet]. 2009 Oct[Cited 2014 May 15]. Available from: http://www.gpo.gov/fdsys/pkg/FR-2009-1001/pdf/E9-23519.pdf

11. Burgunda V S, FASHP, Ann K S, and Dawn M $\mathrm{P}$, Review of the Processes for FDA Oversight of Drugs, Medical Devices, and Combination Products. Journal of Managed Care Pharmacy.
[Internet]. 2011 Jan [Cited 2014 June 24]. Available from:

http://ns.amcp.org/data/jmcp/40-50.pdf 